\title{
Élaboration des cristaux LnCOB pour la conversion de fréquence en accord de phase non critique.
}

\author{
Elisabeth Reino ${ }^{*}$, Gérard Aka**, Jean-Marie Bénitez, Patrick Aschehoug et Daniel Vivien \\ Laboratoire de Chimie Appliquée de l'État solide - ENSCP- UMR 7574 \\ 11, rue P. et M. Curie, F-75231 PARIS CEDEX 05, FRANCE \\ "reino@ext.jussieu.fr, ${ }^{* *}$ akagp@ext.jussieu.fr
}

\begin{abstract}
Résumé. Nous avons étudié différentes substitutions dans le $\mathrm{Ca}_{4} \mathrm{GdO}\left(\mathrm{BO}_{3}\right)_{3}(\mathrm{GdCOB})$ par $\mathrm{Sr}^{2+}, \mathrm{La}^{3+}, \mathrm{Bi}^{3+}, \mathrm{Y}^{3+}, \mathrm{Sc}^{3+}$. L'objectif est de réaliser des dispositifs émettant dans le bleu ou le vert en associant un cristal laser à un cristal non linéaire, le GdCOB «modifié », en accord de phase non critique. Nous avons comparé quelques propriétés de doublage de fréquence à $930 \mathrm{~nm}$ (YAP: $\mathrm{Nd}^{3+}$ ) du $\mathrm{Gd}_{1-\mathrm{x}} \mathrm{Y}_{\mathrm{x}} \mathrm{COB}$ à celles du YCOB en accord de phase « critique » dans les plans $\mathrm{YX}\left(\phi=44,6^{\circ}\right)$ et $\mathrm{ZX}\left(\theta=22,8^{\circ}\right)$.
\end{abstract}

Mots clés. Oxoborates, optique non linéaire, doublage de fréquence, accord de phase non critique, $\mathrm{Gd}_{1-\mathrm{x}} \mathrm{Y}_{\mathrm{x}} \mathrm{COB}, \mathrm{YCOB}$.

\section{INTRODUCTION}

Les besoins industriels ont orienté la recherche vers de nouveaux lasers émettant dans le visible (vert et bleu) ou l'ultraviolet pour des applications telles que le stockage optique de l'information, la médecine, l'affichage sur écran géant, etc. Dans le cas des lasers à l'état solide, la conversion de fréquence apparaît comme une bonne solution en associant des propriétés laser IR et optiques non linéaires (ONL).

En 1992, Norrestam et al. [1] ne mesuraient probablement pas l'engouement que susciterait les oxoborates de lanthanide $\mathrm{LnCa}_{4} \mathrm{O}\left(\mathrm{BO}_{3}\right)_{3}$ ( $\mathrm{LnCOB}$ ), qu'ils venaient de découvrir. En effet, ces matériaux biaxes, relativement non hygroscopiques, stables chimiquement possèdent des propriétés ONL remarquables [2] ayant un intérêt grandissant en particulier pour la conception de sources laser visibles compactes. De plus, ces matériaux à fusion congruente peuvent être élaborés par la méthode de tirage vertical Czochralski. Les cristaux obtenus ont de bonnes propriétés mécaniques, sont transparents dans l'UV et présentent un seuil de dommage optique élevé.

Actuellement, nous cherchons à améliorer les performances des cristaux $\mathrm{GdCa}_{4} \mathrm{O}\left(\mathrm{BO}_{3}\right)_{3}$ (GdCOB) et $\mathrm{YCa}_{4} \mathrm{O}\left(\mathrm{BO}_{3}\right)_{3}$ (YCOB) en conversion de fréquence. La plage de la longueur d'onde utilisable en doublage de fréquence varie en fonction de la biréfringence du matériau. Dans la série $\operatorname{LnCOB}(\mathrm{Ln}=\mathrm{Y}, \mathrm{Gd})$, cette biréfringence, $n_{\mathrm{z}}-n_{\mathrm{x}}$, vaut respectivement 0,042 et 0,033 . Il apparaît donc qu'elle diminue lorsque le rayon ionique augmente. En choisissant une configuration d'orientation simple, telle que l'accord de phase non critique (APNC), i.e. suivant les axes cristallophysiques $X, Y$, $Z$, nous améliorerons l'efficacité de conversion. En effet, lorsque le faisceau laser se propage suivant les axes cristallophysiques nous nous affranchissons des pertes dues à la déviation du faisceau incident car l'angle de double réfraction est nul et l'acceptance angulaire est maximum. Il existe déjà des cristaux permettant l'accès à l'APNC, par exemple pour le doublage de fréquence du YAG:Nd à 1064 $\mathrm{nm}$. C'est le cas pour le $\mathrm{LBO}\left(\mathrm{LiB}_{3} \mathrm{O}_{5}\right)$, mais cela nécessite d'élever la température $\left(\sim 149^{\circ} \mathrm{C}\right)$. Notre ambition est d'obtenir l'APNC, mais à température ambiante, par un ajustement de la composition du GdCOB, celle-ci influant sur la biréfringence. 
Nous décrirons ci-dessous la synthèse, la caractérisation et l'élaboration de monocristaux de ces nouveaux matériaux. Une attention particulière sera portée à la solution solide $\operatorname{Gd}_{1-\mathrm{x}} \mathrm{Y}_{\mathrm{x}} \mathrm{COB}$ en vue du doublage de fréquence d'un laser $\mathrm{YAlO}_{3}: \mathrm{Nd}$ (YAP:Nd) en APNC.

\section{2. ÉLABORATION ET CARACTÉRISATION DES GdCOB SUBSTITUÉS}

Le cas des mixtes $\operatorname{Gd}_{1-x} Y_{x} \mathrm{COB}$ montre que pour la doublage de fréquence les longueurs d'onde fondamentales les plus courtes (du faisceau incident) peuvent varier en type I de $735 \mathrm{~nm}$ (YCOB) à $838 \mathrm{~nm}(\mathrm{GdCOB})$ selon l'axe $Y$ et de $838 \mathrm{~nm}(\mathrm{YCOB})$ à $966 \mathrm{~nm}(\mathrm{GdCOB})$ selon $Z$ [3]]. Il apparaît donc possible d'ajouter cette longueur fondamentale en APNC en préparant des composés mixtes $\mathrm{Gd}_{1-\mathrm{x}} \mathrm{Y}_{\mathrm{x}} \mathrm{COB}$. Mais, l'introduction de scandium, de lutécium, de lanthane, de strontium, permettrait également de contrôler la biréfringence optique.

Une seconde approche nous a conduit à chercher à augmenter le coefficient d'efficacité non linéaire, $d_{\text {eff, }}$ en introduisant des cations présentant une paire d'électrons libres $n s^{2}$ [4] et en espérant que ceux-ci ajouteraient leur polarisabilité à celle des groupements $\left[\mathrm{BO}_{3}\right]^{3-}$ comme pour le $\mathrm{BiBO}\left(\mathrm{BiB}_{3} \mathrm{O}_{6}\right)[\underline{5}]$.

Nous avons donc élaboré les solutions solides : $\mathrm{Gd}_{1-\mathrm{x}} \mathrm{Y}_{\mathrm{x}} \mathrm{COB}, \mathrm{Gd}_{1-\mathrm{x}} \mathrm{La}_{\mathrm{x}} \mathrm{COB}, \mathrm{Gd}_{1-\mathrm{x}} \mathrm{Bi}_{\mathrm{x}} \mathrm{COB}$, $\mathrm{Y}_{1-\mathrm{x}} \mathrm{Bi}_{\mathrm{x}} \mathrm{COB}, \mathrm{Gd}_{1-\mathrm{x}} \mathrm{Sc}_{\mathrm{x}} \mathrm{COB},\left(\mathrm{Ca}_{1-\mathrm{x}} \mathrm{Sr}_{\mathrm{x}}\right)_{4} \mathrm{GdO}\left(\mathrm{BO}_{3}\right)_{3}$.

Les phases choisies sont synthétisées par réaction à l'état solide des oxydes $\left(\mathrm{B}_{2} \mathrm{O}_{3}, \operatorname{Ln}_{2} \mathrm{O}_{3}\right.$ ou $\mathrm{Bi}_{2} \mathrm{O}_{3}$ ) et du carbonate de calcium (ou de strontium) en proportion convenable. Ce mélange est porté à $950{ }^{\circ} \mathrm{C}$ pendant $12 \mathrm{~h}$ (pour la décarbonatation) puis à $1350^{\circ} \mathrm{C}$ pendant $48 \mathrm{~h}$ (formation de la phase). La diffraction des rayons $X$ des composés frittés est utilisée pour vérifier l'insertion des éléments dans la phase souhaitée d'après l'évolution des paramètres de maille.

Nous constatons que le bismuth ne substitue le gadolinium que jusqu'à $30 \%$ dans $\mathrm{Gd}_{1-\mathrm{x}} \mathrm{Bi}_{\mathrm{x}} \mathrm{COB}$ et $\mathrm{Y}_{1-\mathrm{x}} \mathrm{Bi}_{\mathrm{x}} \mathrm{COB}$. Ceci peut s'expliquer d'une part par la « grande » taille de l'ion $\mathrm{Bi}^{3+}$ par rapport à celle de $\mathrm{Gd}^{3+}$ et de $\mathrm{Y}^{3+}$ et d'autre part par l'activité stéréochimique de la paire. En effet, le paramètre $c$ de la maille monoclinique croît plus rapidement que les autres. De même, le rayon ionique du strontium est bien trop important pour que la phase $\mathrm{Sr}_{4} \mathrm{GdO}\left(\mathrm{BO}_{3}\right)_{3}$ soit stable. Sa limite de solubilité dans le GdCOB est de l'ordre de $20 \%$. Le scandium est lui trop petit pour donner une phase ScCOB mais la substitution reste réalisable jusqu'à $60 \%$ au moins. Des essais sont en cours pour $0,6<\mathrm{x}<1$. En revanche, d'autres compositions mixtes forment des solutions à l'état solide complètes. C'est le cas de $\mathrm{Gd}_{1-\mathrm{x}} \mathrm{Y}_{\mathrm{x}} \mathrm{COB}$ et de $\mathrm{Gd}_{1-\mathrm{x}} \mathrm{La}_{\mathrm{x}} \mathrm{COB}$.

L'analyse thermique différentielle (ATD) montre que la plupart des solutions solides possèdent une fusion congruente.

\section{CROISSANCE CRISTALLINE}

Ces dernières mesures nous ont permis d'envisager la cristallogenèse. La faisabilité des cristaux est d'abord vérifiée par la méthode de la fusion de zone. Quelques compositions sont choisies pour un tirage vertical par la méthode Czochralski. Les cristaux obtenus, d'environ $8 \mathrm{~cm}$ de long et de 16 à $20 \mathrm{~mm}$ de diamètre, sont de bonne qualité cristalline et optique. La table I regroupe les paramètres essentiels des croissances réalisées.

De façon générale, ces paramètres ne sont pas optimisés ce qui expliquerait la présence de défauts tels que l'effet de cœur (Fig. 1) ou les faisceaux de bulles parfois associées aux irrégularités du diamètre (i.e. aux variations non voulues et non contrôlées).

La croissance cristalline de $\mathrm{Gd}_{0.28} \mathrm{Y}_{0.72} \mathrm{COB}$, intéressant suivant $Y$ pour le doublage de fréquence de type II et le triplage de fréquence en type I en APNC du laser YAG:Nd à $1064 \mathrm{~nm}$, pose en particulier problème. Après tirage, le cristal se sépare en deux parties : l'une correspondant à la tête et l'autre au corps (Fig. 2). Cette séparation présente concavité sur la fin de la tête. Nous avons d'abord cru à une vitesse de rotation trop élevée, qui aurait pu créer une interface liquide - solide 
TAB. 1. Paramètres de tirage par la méthode Czochralski. Les $T_{\mathrm{F}}$ et $T_{\mathrm{S}}$ désignent les températures de fusion et de solidification observées en ATD. Elles correspondent aux positions des pics au chauffage et au refroidissement à $10^{\circ} \mathrm{C} \cdot \mathrm{min}^{-1}$

\begin{tabular}{|c|c|c|c|c|c|}
\hline Matériaux & $\mathbf{G d}_{0.87} \mathbf{Y}_{0.13} \mathrm{COB}$ & $\mathbf{G d}_{0.84} \mathbf{Y}_{0.16} \mathrm{COB}$ & $\mathbf{G d}_{0.72} \mathbf{Y}_{0.28} \mathrm{COB}$ & $\mathbf{G d}_{0.28} \mathbf{Y}_{0.72} \mathrm{COB}$ & $\mathrm{Gd}_{0.95} \mathrm{Bi}_{0.05} \mathrm{COB}$ \\
\hline $\begin{array}{l}\text { Vitesse de } \\
\text { tirage }\end{array}$ & $1 \mathrm{~mm} \cdot \mathrm{h}^{-1}$ & $2 \mathrm{~mm} \cdot \mathrm{h}^{-1}$ & $0.8 \mathrm{~mm} \cdot \mathrm{h}^{-1}$ & $0.8 \mathrm{~mm} \cdot \mathrm{h}^{-1}$ & $1 \mathrm{~mm} \cdot \mathrm{h}^{-1}$ \\
\hline $\begin{array}{c}\text { Vitesse de } \\
\text { rotation }\end{array}$ & 35 tr.min ${ }^{-1}$ & 16 tr.min $\min ^{-1}$ & 40 tr.min $\min ^{-1}$ & 40 tr.min ${ }^{-1}$ & 35 tr. $\min ^{-1}$ \\
\hline$T_{\mathrm{F}}\left({ }^{\circ} \mathrm{C}\right)$ & 1495 & 1500 & - & 1505 & \\
\hline$T_{\mathrm{S}}\left({ }^{\circ} \mathrm{C}\right)$ & 1315 & 1390 & - & 1370 & \\
\hline
\end{tabular}

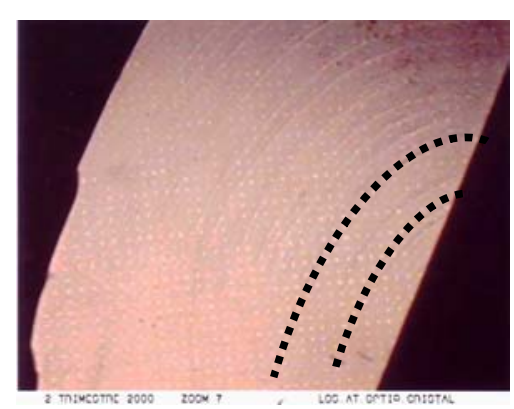

FIG. 1. Effets de cœur dans $\mathrm{Gd}_{0.84} \mathrm{Y}_{0.16} \mathrm{COB}$ (suivant les traits en pointillés et concentriques à l'axe de croissance).

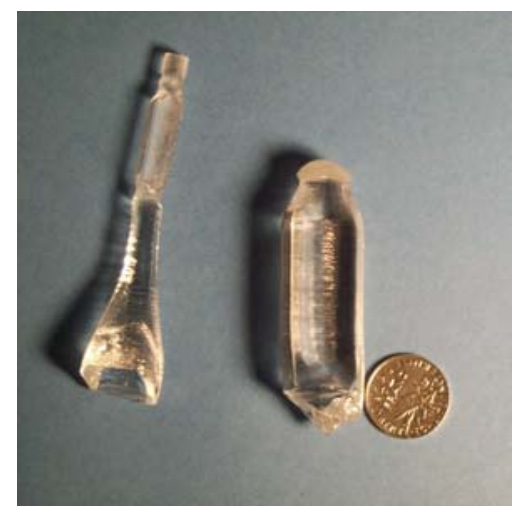

FIG. 2. Cristaux de $\mathrm{Gd}_{0.28} \mathrm{Y}_{0.72} \mathrm{COB}$.

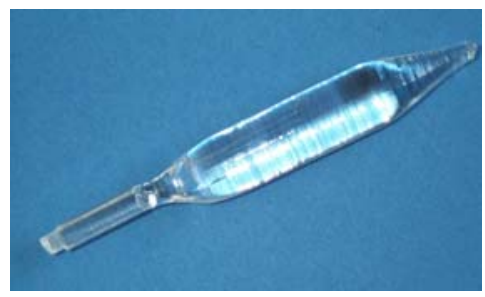

FIG. 3. Cristaux de $\mathrm{Gd}_{0.28} \mathrm{Y}_{0.72} \mathrm{COB}$ dont la composition du fritté possède un excès de $1 \%$ de calcium. 
concave et non plane comme dans le cas idéal []]. En réalité, le ralentissement des rotations n'améliore en rien la qualité. Nous notons également des faisceaux de bulles très fins.

En fait, l'origine de la mauvaise qualité de ce cristal semble provenir du coefficient de ségrégation de 1,12 pour l'yttrium. L'analyse par microsonde électronique indique effectivement une teneur de $84 \%$ de $\mathrm{Y}$ au début du cristal contre $72 \%$ du bain. Nos efforts se poursuivent en but d'améliorer la cristallogenèse : il semblerait que l'ajout de calcium lors de la préparation du fritté (Fig. 3).

\section{4. ÉTUDE COMPARATIVE DES PROPRIÉTÉS DE DOUBLAGE À $930 \mathrm{~nm}$ DU $\mathrm{Gd}_{0.84} \mathrm{Y}_{0.16}$ COB ET DU YCOB}

Le $\mathrm{Gd}_{0.84} \mathrm{Y}_{0.16} \mathrm{COB}$ double la fréquence du YAP:Nd à $930 \mathrm{~nm}$ en type I et en APNC, suivant $Z$ [7]. Nous avons comparé l'efficacité de doublage de fréquence à deux cristaux de YCOB, en accord de phase sur les plans principaux $Y X\left(\phi=44,6^{\circ}\right)$ et $Z X\left(\theta=22,8^{\circ}\right)$. Les pièces utilisées $\left(5^{*} 10^{*} 10 \mathrm{~mm}^{3}\right)$ ont été orientées, taillées et polies. Les premières mesures d'acceptance angulaire sont conformes à nos attentes : pour le YCOB dans le plan $X Y, \Delta \theta \cdot L=1,61 \mathrm{mrad} . \mathrm{cm}$ et dans le plan $Z X$, $\Delta \phi . L=1,35$ mrad.cm alors que pour le GdYCOB $\Delta \theta \cdot L=7,44$ mrad.cm suivant $Z$.

Les mesures d'efficacité de conversion et d'accord de phase seront réalisées prochainement.

\section{CONCLUSION}

Diverses substitutions cationiques ont été réalisées dans le GdCOB pour réajuster la biréfringence d'une part (accès à l'APNC) et d'augmenter le $d_{\text {eff }}$ d'autre part. Les solutions solides partielles $(\mathrm{Bi}, \mathrm{Sr})$ ou totales $(\mathrm{La}, \mathrm{Y})$ et un certain nombre de cristaux ont été élaborés par la méthode Czochralski.

Dans le cas de $\mathrm{Gd}_{0.84} \mathrm{Y}_{0.16} \mathrm{COB}$, adapté au doublage de fréquence du YAP:Nd à $930 \mathrm{~nm}$ en APNC, l'acceptance angulaire s'avère 4 à 5 fois plus élevée que pour les configurations en accord de phase critique à cette même longueur d'onde. Ceci confirme l'intérêt de l'APNC.

\section{REMERCIEMENTS}

Nous remercions Mesdames Jacquet et Lenain (Atelier d'optique cristalline/ Laboratoire d'optique des solides UMR7601-Paris) pour leurs travaux d'orientation, de taille et de polissage, sur l'ensemble de nos cristaux. Merci encore à l'équipe du Pr. D. Keszler et au Dr. A.F.T. Yokochi (Dpt. of Chemistry Oregon State University, Corvallis, USA) de m'avoir aidée et accueillie dans le cadre d'un échange international CNRS-NSF.

\section{RÉFÉRENCES}

[1] R. Norrestam et al., Chem. Mater. 4 (1992) 737-743.

[2] F. Mougel et al., J. Opt. Soc. Am. B 14 (1997) 2238-2247.

[3] M. Iwai et al., Conf. on Laser and Electro-Optics, Technical Digest, CWG2 (1998) 270.

[4] N. Jokubowitz et al., J. Solid State Chem. 139 (1998) 194-199.

[5] H. Hellwig et al., Sol. State Comm. 109, (1999) 249-251.

[6] S. Miyazawa, J. Crystal Growth 49 (1980) 515-521.

[7] P.B.W. Burmester et al., Appl. Phys. B 68 (1999) 1143-1146. 